\author{
Alex Brinkmeyer ${ }^{1}$ \\ Department of Aerospace Engineering, \\ Advanced Composites Centre for \\ Innovation and Science, \\ University of Bristol, \\ Bristol BS8 1TR, UK \\ e-mail: alex.brinkmeyer@bristol.ac.uk \\ Sergio Pellegrino \\ Joyce and Kent Kresa Professor of Aeronautics \\ and Professor of Civil Engineering, \\ Graduate Aerospace Laboratories, \\ California Institute of Technology, \\ Pasadena, CA 91125 \\ e-mail: sergiop@caltech.edu \\ Paul M. Weaver \\ Professor in Lightweight Structures, \\ Department of Aerospace Engineering, \\ Advanced Composites Centre for \\ Innovation and Science, \\ University of Bristol, \\ Bristol BS8 1TR, UK \\ e-mail: paul.weaver@bristol.ac.uk
}

\section{Effects of Long-Term Stowage on the Deployment of Bistable Tape Springs}

In the context of strain-energy-deployed space structures, material relaxation effects play a significant role in structures that are stowed for long durations, for example, in a space vehicle prior to launch. Here, the deployment of an ultrathin carbon fiber reinforced plastic (CFRP) tape spring is studied, with the aim of understanding how longduration stowage affects its deployment behavior. Analytical modeling and experiments show that the deployment time increases predictably with stowage time and temperature, and analytical predictions are found to compare well with experiments. For cases where stress relaxation is excessive, the structure is shown to lose its ability to deploy autonomously. [DOI: 10.1115/1.4031618]

\section{Introduction}

Undesirable time-dependent viscoelastic effects have been previously reported in deployable space structures that are stowed for extended durations prior to launch and subsequent deployment [1]. In thermoset composites, these effects increase as the temperature of the structure approaches the glass transition temperature of the resin. For deployable structures that need to achieve and maintain a precise geometry (e.g., a mirror or radar antenna), it becomes essential to be able to understand and predict the effects of temperature and deformation history on dimensional stability and deployment accuracy [2].

A case in point underlining the significance of time-dependent effects is the Mars Express mission, launched in 2003 by the European Space Agency. This spacecraft, still in operation today, carries thin-walled deployable booms forming the Mars Advanced Radar for Subsurface and Ionosphere Sounding (MARSIS) antenna-designed to search for water on Mars and study its atmosphere. MARSIS is a large closed-section $40 \mathrm{~m}$ antenna manufactured from an S-Glass/Kevlar composite, with periodic slotted sections (Fig. 1). In its stored state, the booms were folded into an accordion manner. When released, the antenna was designed to deploy following a strict latching sequence so as to avoid contact with the spacecraft.

Telemetry flight data from the 2005 deployment of the MARSIS antenna showed two anomalies in its deployment [1]. First, inertial measurements hinted toward the fact that the boom had not completely locked into place. Second, data showed that the deployment dynamics differed significantly from the modeled behavior. The ground testing report noted that a large reduction in the hinge torque was observed, compared to a pristine hinge [1]. The hinge that was tested had undergone multiple thermal cycles and had been stored for $2 \mathrm{yr}$ prior to deployment, as well as being

\footnotetext{
${ }^{\mathrm{I}}$ Corrresponding author.

Contributed by the Applied Mechanics Division of ASME for publication in the Journal of ApPLIEd MeChanics. Manuscript received May 14, 2015; final manuscript received September 15, 2015; published online November 9, 2015. Editor: Yonggang Huang.
}

highly stressed in its stowed configuration. In the final report, the reduction in torque was attributed to significant viscoelastic stress relaxation in the material matrix.

The conclusions from the Mars Express mission report showed that viscoelastic effects can indeed become significant in structures that are stowed for a long time-in this case in a launch vehicle-prior to their deployment in space. It seems therefore crucial to understand precisely how storage affects the deployment behavior of this type of structure.

Another example of a thin-walled deployable boom is the Storable Tubular Extendable Member (STEM), a self-driven, energystoring deployable structure which has gained popularity over the years. The STEM, which resembles a self-extending carpenter's tape measure, was invented in Canada in the 1960s by Rimrott [3] and commercialized a few years later by Astro Aerospace, an affiliate of Northrop Grumman. It has flown successfully on various spacecraft, such as the Voyager probe, the Hubble Space Telescope, and the Mars Pathfinder rover, and is still in production to this day [4].

This paper focuses, in particular, on how storage affects the deployment of ultrathin bistable tape spring booms. In contrast to the STEM, bistable tape springs are designed to be stable both in their coiled and deployed state [3]. As a result, no mechanism is required to maintain the structure in either of these states, thus considerably simplifying the design of the actuator or eliminating the need for it completely. The structures considered herein are inspired by Rolatube's bistable reeled composites, which are commercialized as stowable masts for civil and military applications [5]. They are easily stowed in a backpack and can be readily deployed by hand whenever needed.

Tape springs have been the object of extensive work, in particular by Refs. [6-8], who have studied separate aspects of deployment dynamics, bistability, and viscoelastic shape recovery. This paper aims to reconcile these three aspects and provide a better understanding of time-dependent effects in bistable tape spring structures. Building on a preliminary study by the present authors [9], this paper seeks to enhance the understanding of long-term stowage effects in such structures. The objectives are first to 


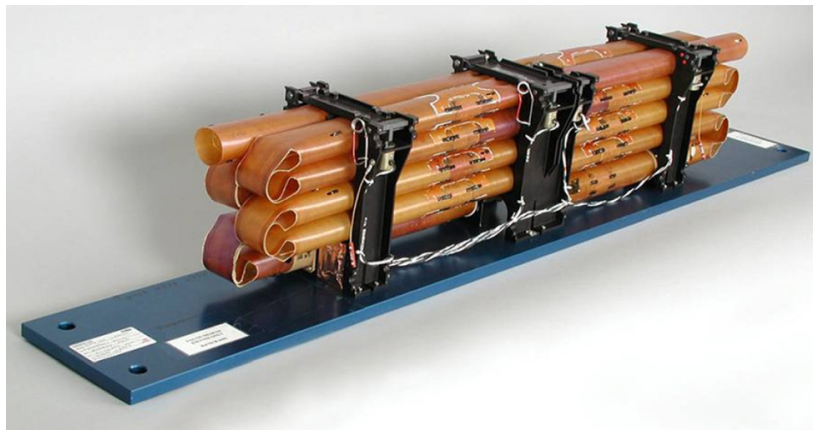

Fig. 1 MARSIS antenna boom structure. The boom consists of a single tubular structure with several flatennable slotted sections which act as elastic hinges (courtesy of Astro Aerospace).
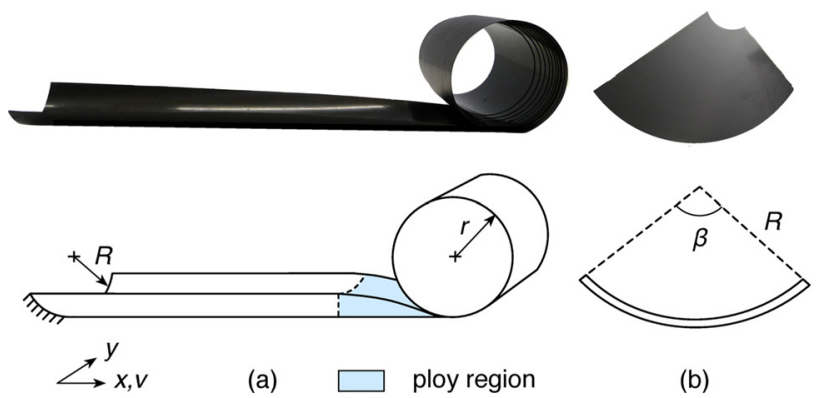

(b)

Fig. 2 Bistable tape spring geometry with (a) the side view of partially coiled spring, $(b)$ the front view of fully deployed spring; $r$ is the coiled radius and $R$ is the deployed radius, with $\beta$ the subtended angle of the deployed spring

investigate the change of deployment speed with different stowage conditions (time and temperature) using an analytical model and experimental tests and second, to understand how the stability of bistable tape springs changes with stowage time.

This paper is structured as follows. The geometry of the tape spring and the stowage model are first described. The stability of the tape spring is then analyzed using an analytical model and verified using lamination parameters. The deployment of the tape spring is investigated for various stowage conditions and compared with experimental tests. Finally, singular deployment cases are discussed before conclusions are drawn.

\section{Geometry and Material Definition}

The tape, in its partially and fully deployed configuration, is shown in Fig. 2. The geometry of the coiled structure is characterized by the radius of coiling $r$, while the extended configuration has transverse radius $R$, subtends an angle $\beta$, and has a total length $L$. The transition between coiled and uncoiled regions of the tape spring is called ploy region. It has no significant effect on the deployment behavior and is neglected throughout this paper. The measured geometrical parameters are given in Table 1 [10].

The material selected for the prototype structures that are studied in this paper is ThinPly $T 800 \mathrm{H}$, an ultrathin unidirectional carbon fiber prepreg produced by North Thin Ply Technology. The ply thickness was found experimentally to be $30 \pm 1 \mu \mathrm{m}$, using ultrasonic measurement of cured $[0]_{6}$ and $[90]_{6}$ laminates (the subscript denotes the number of plies in usual composite notation). Additionally, the material elastic properties at room temperature were determined experimentally using an Instron materials testing machine and a stereoscopic digital image correlation (DIC) Correlated Solutions imaging system. These properties are given in Table 2.
Table 1 Tape spring geometrical parameters

\begin{tabular}{lccc}
\hline \hline$R(\mathrm{~mm})$ & $r(\mathrm{~mm})$ & $\beta(\mathrm{deg})$ & $L(\mathrm{~mm})$ \\
\hline 16.6 & 20.8 & 131.8 & 1253 \\
\hline \hline
\end{tabular}

Table 2 T800H elastic properties at room temperature [10]

\begin{tabular}{lccc}
\hline \hline$E_{11}(\mathrm{GPa})$ & $E_{22,0}(\mathrm{MPa})$ & $G_{12,0}(\mathrm{MPa})$ & $\nu_{12}$ \\
\hline 128 & 6.5 & 7.6 & 0.35 \\
\hline \hline
\end{tabular}

\section{STOWAGE}

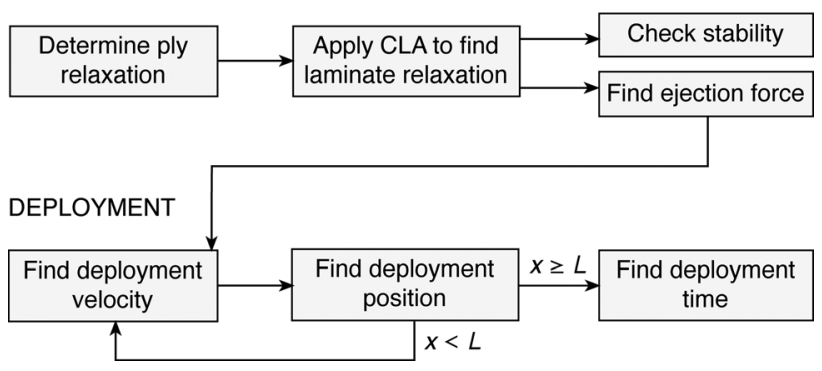

Fig. 3 Flow diagram of the tape spring analytical models

The antisymmetric layup [45/-45/0/45/-45] is used throughout, with the 0 deg direction oriented along the length of the tape spring. The layup is chosen to be antisymmetric as this eliminates bend-twist effects during coiling, which would otherwise exist in a symmetric layup because of flexural/twist anisotropy.

The tape spring, manufactured in its extended shape, is fully coiled and stored at a given temperature. Before any deployment can occur, one end of the coil is snapped out and fixed in place. This procedure is necessary to start the deployment process, because the structure is fully stable in its coiled state. The other end of the structure is then allowed to deploy autonomously. The position of the center of the coil is then measured with time. The analytical model consists of three parts:

(1) Stowage model: Transforms the material relaxation data obtained at ply level to the overall laminate relaxation using classical laminate analysis (CLA) [11].

(2) Stability model: Essentially a submodel of the stowage model, the stability model simulates the nature of the structure's stability during the stowage process.

(3) Deployment model: Uses the material state at the end of the stowage process to predict the dynamic deployment of the tape spring.

A synoptic flow diagram of the models is given in Fig. 3. The models span two distinctively different time scales-the stowage and stability models simulate behavior over several hours or days, while the deployment model predicts dynamic behavior occurring in fractions of a second. The outputs of the stowage model (in particular, the net ejection force) are used as inputs to the deployment model. Sections 3-6 focus on each model in detail and present the results obtained.

\section{Stowage Material Model}

3.1 Characterization of Ply Relaxation. The carbon fibers are assumed to be elastic for the observed relaxation times and temperatures. Hence, because the deformation in the longitudinal 1-direction is dominated by the fibers, it is assumed that the Young's modulus of the composite lamina in the 1-direction is constant, $E_{11}(t)=E_{11}$. 


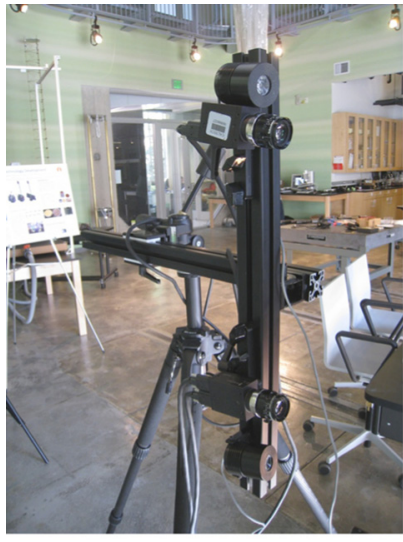

(a)

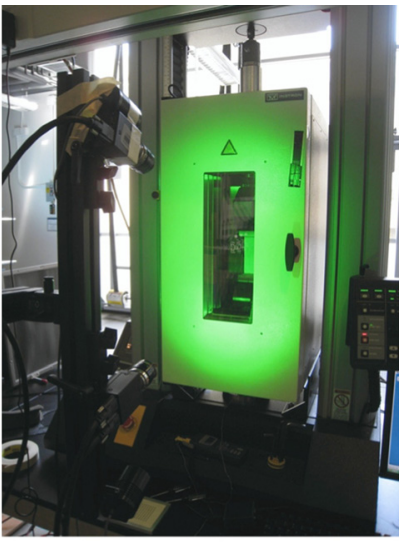

(b)
Fig. 4 Relaxation experimental apparatus, consisting of: (a) a DIC imaging system and $(b)$ a thermal chamber mounted inside an Instron testing machine

Stress relaxation tests on $[90]_{6}$ laminates were performed to determine the viscoelastic behavior in transverse tension $E_{22}(t)$. An Instron 5500 Series machine equipped with a thermal chamber and a thermocouple was used for the relaxation tests (Fig. 4). The specimen and the Instron grips were warmed to achieve thermal equilibrium prior to starting the relaxation test. The Instron crosshead displacement was then adjusted to remove any slack induced by temperature effects, and the specimen was allowed to rest for another $30 \mathrm{~min}$. The transient thermal expansion of the rig was found to have a significant influence on the relaxation results, as thermal expansion strains were of the same order of magnitude as the mechanical strains. Hence, achieving thermal equilibrium prior to the test was crucial for accurate results.

The relaxation test consisted of applying a quasi-instantaneous strain of $\varepsilon_{11,0}=0.1 \%$ to the specimen and holding this value for $3 \mathrm{hrs}$. The load and strain were recorded, respectively, through the Instron machine load cell and the 3D DIC system. It was found that the calculation of the instantaneous modulus $E_{22.0}$ from the relaxation test was inaccurate because of the small strains involved. Hence, a separate tension test was used to determine $E_{22,0}$ at each temperature to complete the characterization of the material.

A Prony series is chosen to represent the relaxation modulus in the transverse direction

$$
E_{22}(t)=E_{22,0}\left(1-\sum_{i=1}^{N} e_{\mathrm{R} i}\left(1-e^{-t / \tau_{i}}\right)\right)
$$

where $e_{\mathrm{R} i}$ are the tensile relaxation coefficients associated with the transverse modulus $E_{22}(t)$, and $\tau_{i}$ are the characteristic times. A similar expression can be defined for the shear modulus, where $g_{\mathrm{R} i}$ are the shear relaxation coefficients associated with the shear modulus $G_{12}(t)$. It is assumed that the material has a timeindependent Poisson's ratio, hence it is possible to set $e_{\mathrm{R} i}=g_{\mathrm{R} i}$.

The Prony series is fitted to the experimental data curves using a bespoke [check] MATLAB nonlinear optimization algorithm using the fminsearch function [12]. Starting from an initial estimate of the Prony series coefficients, this algorithm converges to the final coefficients that minimize the least squares error.

Figure 5 shows the fitting of the normalized transverse modulus $E_{22}(t) / E_{22,0}$ for stowage at $60^{\circ} \mathrm{C}$. The Prony series coefficients are listed in Table 3. It was found that four terms were sufficient to provide an accurate nonlinear fitting, by minimization of the fit error. Additionally, fitting of the curve is adjusted so that the normalized modulus is equal to 1 at the start of the test.

Figure 5 shows that at this temperature the material experiences a significant drop in stiffness, decreasing by around 35\% after

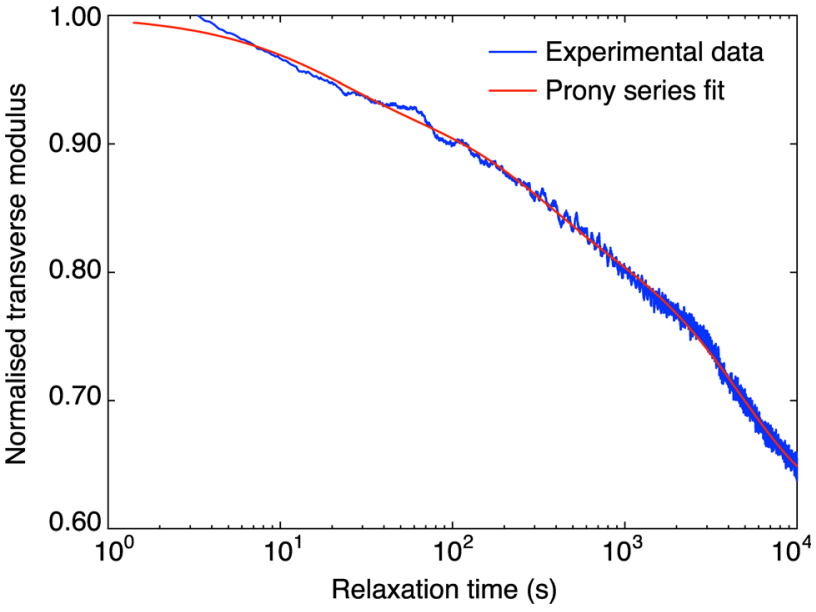

Fig. 5 Normalized transverse modulus fitted with a Prony series for relaxation at $60^{\circ} \mathrm{C}$ for $3 \mathrm{hrs}$

Table 3 Prony series coefficients for relaxation at $60^{\circ} \mathrm{C}$ for $3 \mathrm{hrs}$

\begin{tabular}{lcc}
\hline \hline $\begin{array}{l}\text { Term } \\
i\end{array}$ & $e_{\mathrm{R} i}$ & $\begin{array}{c}\tau_{i} \\
(\mathrm{~s})\end{array}$ \\
\hline 1 & 0.013 & 2.64 \\
2 & 0.062 & 16.3 \\
3 & 0.094 & 266 \\
4 & 0.219 & 4790 \\
\hline \hline
\end{tabular}

$3 \mathrm{hrs}$. Oscillations were found to appear in the relaxation data, growing in amplitude with increasing temperature. These fluctuations are due to periodic variations in temperature inside the thermal chamber, as the heating element turns on and off repeatedly. Because the period of these oscillations was much smaller than the total test time, no significant effect on the results was found. Presmoothing of the data prior to fitting the Prony series was not deemed to be necessary.

In this paper, it is sufficient to consider relaxation at $60^{\circ} \mathrm{C}$ as it gives an indication of how the structure behaves at a higher temperature, compared to room temperature. The epoxy resin used herein can be assumed to be rheologically simple, meaning that time and temperature effects can be used interchangeably to describe the behavior of the material under relaxation. Using the principle of time-temperature superposition, testing at a higher temperature is equivalent to reducing the relaxation time of the material [13]. Obtaining the relaxation curves at two other temperatures would be required to complete the characterization of the material, but this is beyond the aim of this paper.

3.2 Transformation to Laminate Relaxation. The lamina stiffnesses defined in Sec. 3.1 are transformed to laminate stiffnesses using CLA. In the CLA theory, the ABD matrix represents the laminate stiffness matrix relating in-plane strains $\varepsilon^{0}$ and curvatures, $\kappa$, to in-plane stress $\mathbf{N}$ and bending moment $\mathbf{M}$, resultants [14]

$$
\left[\begin{array}{l}
N_{x x} \\
N_{y y} \\
N_{x y} \\
\hline M_{x x} \\
M_{y y} \\
M_{x y}
\end{array}\right]=\left[\begin{array}{lll|lll}
A_{11} & A_{12} & A_{16} & B_{11} & B_{12} & B_{16} \\
A_{12} & A_{22} & A_{26} & B_{12} & B_{22} & B_{26} \\
A_{16} & A_{26} & A_{66} & B_{16} & B_{26} & B_{66} \\
\hline B_{11} & B_{12} & B_{16} & D_{11} & D_{12} & D_{16} \\
B_{12} & B_{22} & B_{26} & D_{12} & D_{22} & D_{26} \\
B_{16} & B_{26} & B_{66} & D_{16} & D_{26} & D_{66}
\end{array}\right]\left[\begin{array}{c}
\varepsilon_{x x}^{0} \\
\varepsilon_{y y}^{0} \\
\varepsilon_{x y}^{0} \\
\hline \kappa_{x x} \\
\kappa_{y y} \\
\kappa_{x y}
\end{array}\right]
$$




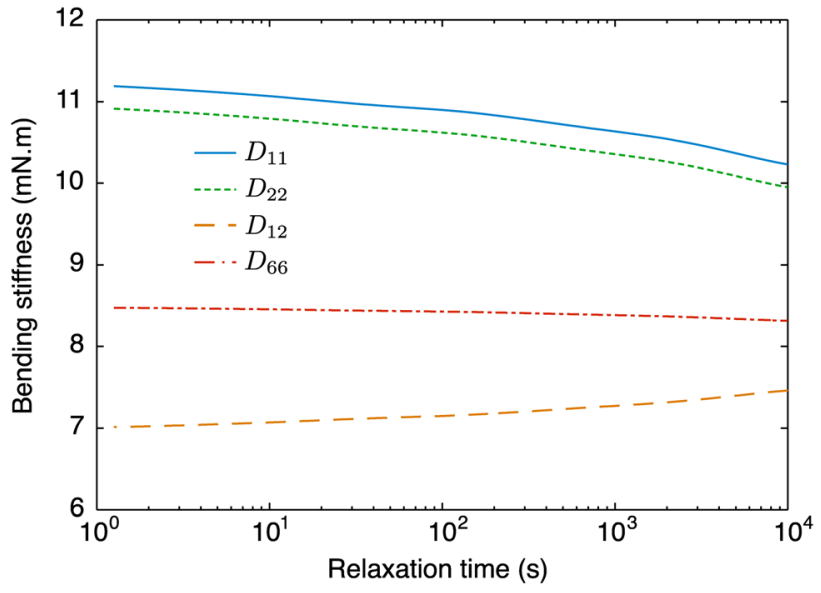

Fig. 6 Evolution of the bending stiffnesses $D_{i j}$ for relaxation at $60^{\circ} \mathrm{C}$. The $D_{11}, D_{22}$, and $D_{66}$ terms decrease with relaxation time, while the $D_{12}$ term increases. The $D_{16}$ and $D_{26}$ components are zero and are therefore not shown.

The [45/-45/0/45/-45] layup considered herein is balanced, as it has equal number of positive and negative angled plies. As a consequence, $A_{16}=A_{26}=0$, i.e., the layup has zero extensionshear coupling. Because the layup is also clearly antisymmetric, the bend-twist components $D_{16}$ and $D_{26}$ are zero, resulting in a state of flexural orthotropy. However, the antisymmetry induces a small coupling between the extension and bending components $B_{16}$ and $B_{26}$.

As the material is viscoelastic, the laminate's ABD matrix is not a constant material property and changes with time as the material relaxes. The four computed nonzero components of the laminate bending stiffness (D matrix) are plotted in Fig. 6 with relaxation time, and the plot shows that $D_{11}, D_{22}$, and $D_{66}$ decrease with relaxation time, while $D_{12}$ increases. (The $D_{16}$ and $D_{26}$ components are zero and therefore are not shown.) Relaxation causes a relatively large decrease of the longitudinal bending stiffness, with the $D_{11}$ and $D_{22}$ terms each decreasing by approximately $9 \%$. On the other hand, the decrease in torsional stiffness $D_{66}$ was found to be negligible.

\section{Stability Analysis}

4.1 Stability Model. Elastic bistable structures contain two energy "wells" or minima in their elastic potential [15], with a tailorable deployment required to trigger the transition between these stable configurations. Therefore, no external force is needed to maintain the structure in each of its stable configurations. Such behavior is highly desirable for deployable structures. A bistable tape spring, for example, rests in either of two stable configurations - coiled and fully deployed.

On the other hand, for a time-dependent structure, such conclusions can no longer be drawn because viscoelastic bistable structures do not have a uniquely defined potential surface [15]. To determine whether the structure studied herein remains bistable with time, a criterion of stability for cylindrical shells, $S$, is computed during relaxation.

$$
S=4 \hat{D}_{66}+2 \hat{D}_{12}-2 \frac{\hat{D}_{22}}{\hat{D}_{12}}
$$

where $\hat{\mathbf{D}}$ is the nondimensional bending stiffness, given by $\hat{\mathbf{D}}=\mathbf{D} / D_{11}$.

Previous research showed that, for elastic bistable structures, the existence of the second stable equilibrium depends only on the sign of Eq. (3) [16]. When $S>0$, the tape spring is bistable, and when $S \leq 0$ the structure is monostable. Larger torsional stiffness
$\hat{D}_{66}$ and anticlastic stiffness $\hat{D}_{12}$ tend to increase the bistability of the spring, while a larger transverse stiffness $\hat{D}_{22}$ tends to decrease it.

It is proposed that the stability criterion is adapted to timedependent bistable structures; by allowing the nondimensional constants to vary with time, $S$ is computed at every point in time during relaxation. Additionally, a reduced bending stiffness matrix $\mathbf{D}^{*}$ is introduced to correct for the nonzero $\mathbf{B}$ coupling matrix. Hence, the stability criterion is now written as

$$
S=4 \hat{D}_{66}^{*}(t)+2 \hat{D}_{12}^{*}(t)-2 \frac{\hat{D}_{22}^{*}(t)}{\hat{D}_{12}^{*}(t)}
$$

where the reduced bending stiffness is given by $\mathbf{D}^{*}=\mathbf{D}$ $-\mathbf{B} \mathbf{A}^{-1} \mathbf{B}$.

4.2 Stability Results. The nondimensional reduced $\hat{\mathbf{D}}^{*}$ matrix at room temperature and at the start of relaxation is given, for the structure considered, by

$$
\hat{\mathbf{D}}^{*}=\left[\begin{array}{ccc}
1 & 0.607 & 0 \\
0.607 & 0.976 & 0 \\
0 & 0 & 0.745
\end{array}\right]
$$

Thus, using Eq. (4), the stability criterion at time $t=0$ is calculated to be $S(0)=0.95$, which is a positive value with a suitable margin of stability. Therefore, it can be concluded that the $[45 /-45 / 0 / 45 /-45]$ layup yields a suitably bistable structure.

The variation of the three nondimensional parameters composing $S$, i.e., $\hat{D}_{12}^{*}, \hat{D}_{22}^{*}, \hat{D}_{66}^{*}$, is now investigated. These terms are plotted in Fig. 7 for the case of stowage $60^{\circ} \mathrm{C}$ for $3 \mathrm{hrs}$. The plot shows that, contrary to the stiffnesses represented in Fig. 6, both $\hat{D}_{12}^{*}$ and $\hat{D}_{66}^{*}$ increase with relaxation time, while $\hat{D}_{22}^{*}$ remains approximately constant.

By inspecting each term of Eq. (3) separately, the two positive terms increase while the negative term decreases; therefore, $S$ increases with relaxation time. Plotting the stability criterion against the relaxation time in Fig. 8 does show that $S$ increases from $S=1.20$ at the start of the relaxation to $S=2.05$ for a relaxation time of $t=3 \mathrm{hrs}\left(t \approx 10^{4} \mathrm{~s}\right)$.

It is noted that the behavior shown in Fig. 8 is representative of a general stowage case; therefore, conclusions can be drawn for any stowage time and temperature. Using the principle of time-temperature superposition, then provided that $S(0)>0$ at the lower bound of temperatures studied, then $S(0)>0$ for any higher temperature [13]. The unequivocal increase of the stability

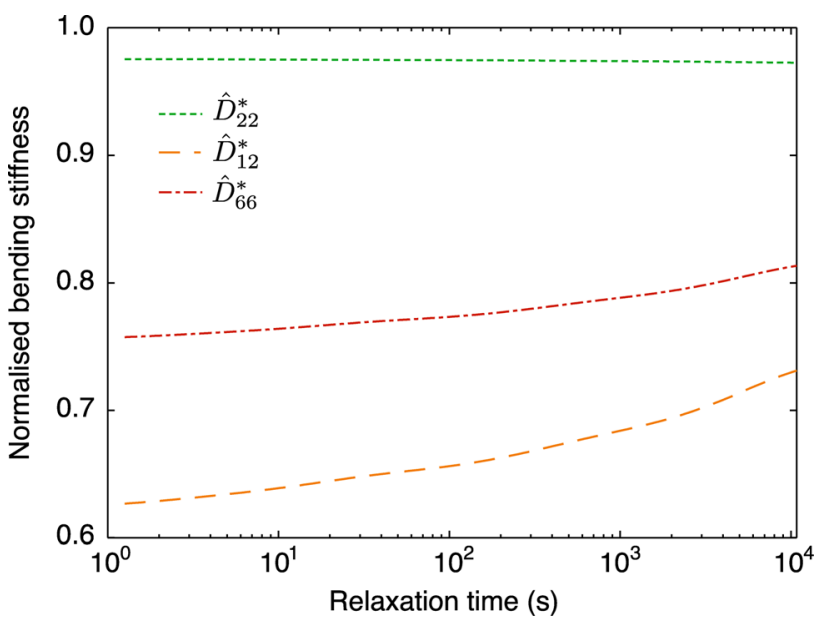

Fig. 7 Evolution of the nondimensional parameters for relaxation at $60^{\circ} \mathrm{C}$. The $\hat{D}_{12}^{*}$ and $\hat{D}_{66}^{*}$ terms increase with relaxation time, while $\hat{D}_{22}^{*}$ remains approximately constant. 


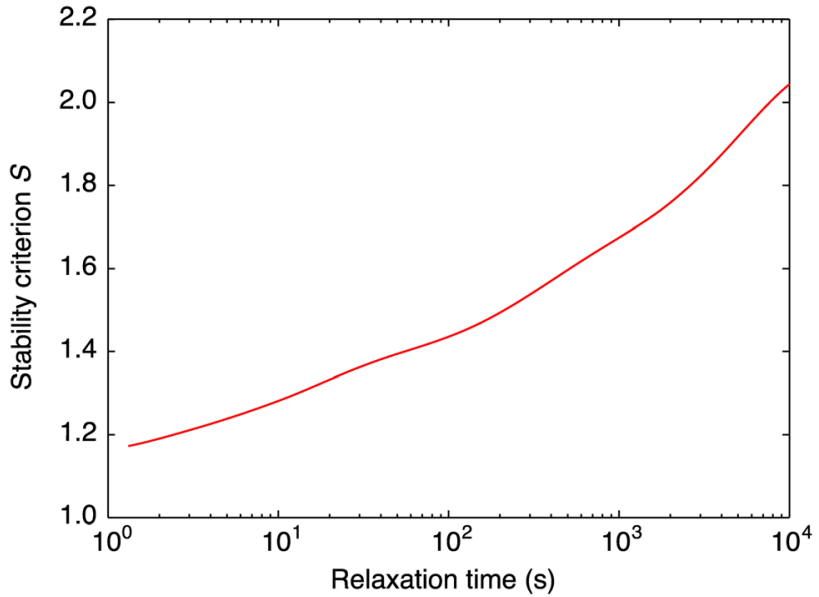

Fig. 8 Evolution of the stability criterion for relaxation at $60^{\circ} \mathrm{C}$. $S$ increases monotonically with time, which suggests that the tape spring remains bistable and does not autonomously unfurl.

criterion with relaxation time leads to an important conclusionprovided the structure is initially bistable, it will remain so during the relaxation process. In other words, the tape spring will not self-deploy once it is stored in its coiled state, even if it is unconstrained.

\section{Validation of the Stability Analysis}

5.1 Flexural Stiffness Analysis. The previous stability analysis is now validated using a different analytical technique. The flexural stiffness $\mathbf{D}$ can be represented by a linear combination of material invariant stiffnesses, stacking sequence effects described by lamination parameters $\xi^{\mathbf{D}}$, and second moment of area by

$$
\mathbf{D}=\frac{h^{3}}{12} \boldsymbol{\Gamma} \cdot \xi^{\mathbf{D}}
$$

where

$$
\xi^{\mathbf{D}}=\left[\begin{array}{lllll}
1 & \xi_{1}^{D} & \xi_{2}^{D} & \xi_{3}^{D} & \xi_{4}^{D}
\end{array}\right]^{\mathrm{T}}
$$

and

$$
\xi_{1,2,3,4}^{D}=\frac{12}{h^{3}} \int_{-h / 2}^{h / 2}(\cos 2 \theta, \quad \cos 4 \theta, \quad \sin 2 \theta, \quad \sin 4 \theta) z^{2} \mathrm{~d} z
$$

where $z$ is the distance to the midplane, and $h$ is the total laminate thickness [17]. The material property matrices vector $\Gamma$ is defined as

$$
\boldsymbol{\Gamma}=\left[\begin{array}{lllll}
\boldsymbol{\Gamma}_{0} & \boldsymbol{\Gamma}_{1} & \boldsymbol{\Gamma}_{2} & \boldsymbol{\Gamma}_{3} & \boldsymbol{\Gamma}_{4}
\end{array}\right]
$$

where

$$
\begin{aligned}
& \boldsymbol{\Gamma}_{0}= {\left[\begin{array}{ccc}
W_{1} & W_{4} & 0 \\
W_{4} & W_{1} & 0 \\
0 & 0 & W_{5}
\end{array}\right] ; \quad \boldsymbol{\Gamma}_{1}=\left[\begin{array}{ccc}
W_{2} & 0 & 0 \\
0 & -W_{2} & 0 \\
0 & 0 & 0
\end{array}\right] } \\
& \boldsymbol{\Gamma}_{2}= {\left[\begin{array}{ccc}
W_{3} & -W_{3} & 0 \\
-W_{3} & W_{3} & 0 \\
0 & 0 & -W_{3}
\end{array}\right] ; \quad \boldsymbol{\Gamma}_{3}=\frac{1}{2}\left[\begin{array}{ccc}
0 & 0 & W_{2} \\
0 & 0 & W_{2} \\
W_{2} & W_{2} & 0
\end{array}\right] } \\
& \boldsymbol{\Gamma}_{4}=\left[\begin{array}{ccc}
0 & 0 & W_{3} \\
0 & 0 & -W_{3} \\
W_{3} & -W_{3} & 0
\end{array}\right]
\end{aligned}
$$

Here, $W_{i}$ are the material invariants defined as

$$
\begin{aligned}
W_{1} & =\frac{1}{8}\left(3 Q_{11}+3 Q_{22}+2 Q_{12}+4 Q_{66}\right) \\
W_{2} & =\frac{1}{2}\left(Q_{11}-Q_{22}\right) \\
W_{3} & =\frac{1}{8}\left(Q_{11}+Q_{22}-2 Q_{12}-4 Q_{66}\right) \\
W_{4} & =\frac{1}{8}\left(Q_{11}+Q_{22}+6 Q_{12}-4 Q_{66}\right) \\
W_{5} & =\frac{1}{8}\left(Q_{11}+Q_{22}-2 Q_{12}+4 Q_{66}\right)
\end{aligned}
$$

where $Q_{i}$ are the lamina stiffnesses in the 1-2 material coordinate system given by

$$
\begin{array}{ll}
Q_{11}=\frac{E_{11}}{1-\nu_{12} \nu_{21}} ; & Q_{12}=\frac{\nu_{12} E_{22}}{1-\nu_{12} \nu_{21}} \\
Q_{22}=\frac{E_{22}}{1-\nu_{12} \nu_{21}} ; & Q_{66}=G_{12}
\end{array}
$$

and $E_{11}, E_{22}, G_{12}, \nu_{21}$, and $\nu_{21}$ are the basic ply stiffness properties.

5.2 Application to Tape Spring Problem. The [45/-45/0/ 45/-45] laminate considered herein is balanced, antisymmetric, and contains a combination of $0 \mathrm{deg}$ and $\pm 45 \mathrm{deg}$ plies only. Therefore by inspection of the trigonometric terms, $\xi_{4}^{D}=0$. Additionally, as has already been shown, $D_{16}=D_{26}=0$ due to the layup asymmetry; therefore,

$$
\begin{aligned}
& D_{11}=\frac{h^{3}}{12}\left(W_{1}+\xi_{1}^{D} W_{2}+\xi_{2}^{D} W_{3}\right) \\
& D_{22}=\frac{h^{3}}{12}\left(W_{1}-\xi_{1}^{D} W_{2}+\xi_{2}^{D} W_{3}\right) \\
& D_{12}=\frac{h^{3}}{12}\left(W_{4}-\xi_{2}^{D} W_{3}\right) \\
& D_{66}=\frac{h^{3}}{12}\left(W_{5}-\xi_{2}^{D} W_{3}\right)
\end{aligned}
$$

The outer \pm 45 deg plies of the laminate contribute to most of the bending stiffness, due to their enhanced second moment of area contribution. To simplify the analysis the inner 0 deg ply can therefore be neglected. Thus the lamination parameters further simplify to

$$
\xi_{1}^{D} \approx 0 ; \quad \xi_{2}^{D} \approx-1
$$

(A full analysis reveals in fact that $\xi_{1}^{D}=0.008$ and $\xi_{2}^{D}=-0.984$; hence, the above simplification is valid.) Thus, Eq. (13) reduces to

$$
\begin{aligned}
& D_{11} \approx \frac{h^{3}}{12}\left(W_{1}-W_{3}\right) \\
& D_{22} \approx \frac{h^{3}}{12}\left(W_{1}-W_{3}\right) \\
& D_{12} \approx \frac{h^{3}}{12}\left(W_{3}+W_{4}\right) \\
& D_{66} \approx \frac{h^{3}}{12}\left(W_{3}+W_{5}\right)
\end{aligned}
$$

It is noted that with this assumption $D_{11} \approx D_{22}$. Observing Fig. 6, it can be noticed indeed that the $D_{11}$ and $D_{22}$ curves follow closely. $D_{11}$ remains slightly larger than $D_{22}$ because of the bending orthotropy introduced by the middle 0 deg ply. 
Substituting the material invariants $W_{i}$ from Eq. (11) and simplifying, the following expressions are obtained

$$
\begin{aligned}
& D_{11} \approx \frac{h^{3}}{48}\left(Q_{11}+Q_{22}+2 Q_{12}+4 Q_{66}\right) \\
& D_{22} \approx \frac{h^{3}}{48}\left(Q_{11}+Q_{22}+2 Q_{12}+4 Q_{66}\right) \\
& D_{12} \approx \frac{h^{3}}{48}\left(Q_{11}+Q_{22}+2 Q_{12}-4 Q_{66}\right) \\
& D_{66} \approx \frac{h^{3}}{48}\left(Q_{11}+Q_{22}-2 Q_{12}\right)
\end{aligned}
$$

5.3 Variational Viscoelastic Analysis. Let us now consider the variation of the $D_{i j}$ coefficients with time. While it is impractical to define a closed-form viscoelastic expression, it is possible to study instead the bounding states of the material-relaxed and unrelaxed. These bounds are denoted by subscripts " $U$ " for the unrelaxed state and by "R" for the fully relaxed state. The assumptions stated previously are summarized as follows:

(1) The longitudinal Young's modulus $E_{11}$ does not change with time. Due to its molecular composition, carbon fiber experiences negligible creep. Using the rule of mixtures in the longitudinal direction, this justifies why $E_{11}$ can be assumed constant.

(2) The Poisson's ratio $\nu_{12}$ is time-independent.

(3) The relaxation causes a decrease of the shear modulus and Young's modulus with time, i.e., $G_{12, \mathrm{R}}<G_{12, \mathrm{U}}$ and $E_{22, \mathrm{R}}<E_{22, \mathrm{U}}$. This is a physical process dominated by the relaxation mechanism of the epoxy resin, whereby applying a strain to the material causes a decrease in its apparent modulus [13].

(4) The relaxation of the shear modulus and Young's modulus occurs at the same rate, i.e., $G_{12, \mathrm{R}} / G_{12, \mathrm{U}}=E_{22, \mathrm{R}} / E_{22, \mathrm{U}}$.

For simplicity, yet without losing qualitative understanding, the B matrix coupling terms are ignored; therefore, it can be assumed, with little loss of accuracy that $D_{i j}^{*}=D_{i j}$.

Using these assumptions, it is possible to draw immediate conclusions concerning Eq. (16). Assumption 1 means that $Q_{11}$ is constant with time. Additionally, assumptions 2 and 3 together show that $Q_{12}, Q_{22}$, and $Q_{66}$ decrease with time. Therefore, overall $D_{11}$ and $D_{22}$ decrease with time, which is consistent with the behavior of the curves in Fig. 6.

On the other hand, the $D_{12}$ and $D_{66}$ terms do not lead to such an immediate conclusion. To understand this phenomenon better, the relative contribution of each term is examined. Substituting the material constants $Q_{i j}$ from Eq. (12), the $D_{12}$ term is written as

$$
D_{12}=\frac{h^{3}}{48}\left(\frac{E_{11}}{1-\nu_{12} \nu_{21}}+\frac{E_{22}}{1-\nu_{12} \nu_{21}}\left(1+2 \nu_{12}\right)-4 G_{12}\right)
$$

Similarly, $D_{66}$ is expanded as

$$
D_{66}=\frac{h^{3}}{48}\left(\frac{E_{11}}{1-\nu_{12} \nu_{21}}+\frac{E_{22}}{1-\nu_{12} \nu_{21}}\left(1-2 \nu_{12}\right)\right)
$$

For the materials considered herein $1-2 \nu_{12}>0$ as $0<\nu_{12}<0.5$. Therefore, by inspection the $D_{66}$ term also decreases with relaxation.

To establish whether $D_{12}$ increases with relaxation, let us study the sign of $D_{12, \mathrm{R}}-D_{12, \mathrm{U}}$.

$$
\begin{aligned}
D_{12, \mathrm{R}} & -D_{12, \mathrm{U}} \\
= & \frac{h^{3}}{48}\left[E_{22, \mathrm{U}}\left(\frac{E_{22, \mathrm{R}}}{E_{22, \mathrm{U}}}-1\right)\left(\frac{1+2 \nu_{12}}{1-\nu_{12} \nu_{21}}\right)-4 G_{12, \mathrm{U}}\left(\frac{G_{12, \mathrm{R}}}{G_{12, \mathrm{U}}}-1\right)\right]
\end{aligned}
$$

Using assumption 4 and rearranging then

$$
\begin{aligned}
D_{12, \mathrm{R}} & -D_{12, \mathrm{U}} \\
= & \frac{h^{3}}{48}\left[\left(\frac{E_{22, \mathrm{R}}}{E_{22, \mathrm{U}}}-1\right)\left[E_{22, \mathrm{U}}\left(\frac{1+2 \nu_{12}}{1-\nu_{12} \nu_{21}}\right)-4 G_{12, \mathrm{U}}\right]\right]
\end{aligned}
$$

Because $E_{22, \mathrm{R}} / E_{22, \mathrm{U}}<1$ then

$$
\frac{E_{22, \mathrm{R}}}{E_{22, \mathrm{U}}}-1<0
$$

therefore the sign of $D_{12, \mathrm{R}}-D_{12, \mathrm{U}}$ depends on the sign of the second term. Thus, $D_{12, \mathrm{R}}-D_{12, \mathrm{U}}>0$ if, and only if,

$$
E_{22, \mathrm{U}}\left(\frac{1+2 \nu_{12}}{1-\nu_{12} \nu_{21}}\right)-4 G_{12, \mathrm{U}}<0
$$

leading to the relation

$$
\frac{E_{22, \mathrm{U}}}{G_{12, \mathrm{U}}}<\frac{4\left(1-\nu_{12} \nu_{21}\right)}{1+2 \nu_{12}}
$$

With $\nu_{12}=0.35$, Eq. (23) becomes

$$
\frac{E_{22, \mathrm{U}}}{G_{12, \mathrm{U}}}<2.34 z
$$

The transverse Young's modulus needs to be more than twice as high as the shear modulus for this expression to hold. Here, at room temperature

$$
\frac{E_{22, \mathrm{U}}}{G_{12, \mathrm{U}}}=\frac{6.5}{7.6}=0.86
$$

and so verifying Eq. (23), which means that

$$
D_{12, \mathrm{R}}-D_{12, \mathrm{U}}>0
$$

thus proving the unusual result that $D_{12}$ increases with relaxation for the material considered. It is worth noting that Eq. (23) is conveniently expressed in terms of unrelaxed values only; in other words, this relation only depends on the initial material properties.

5.4 Analysis of Dimensionless Terms. To conclude this analytical study, the nondimensional terms composing $S$ are now studied. First of all, as has previously been shown, $D_{12}$ increases while $D_{11}$ decreases with time, so it can be concluded immediately that $\hat{D}_{12}=D_{12} / D_{11}$ must rise with relaxation time. Moreover, using Eq. (15) then

$$
\hat{D}_{22} \approx \frac{W_{1}-W_{3}}{W_{1}-W_{3}}=1
$$

Hence $\hat{D}_{22}$ remains constant with relaxation. Finally, studying the $\hat{D}_{66}$ term

$$
\hat{D}_{66} \approx \frac{W_{3}+W_{5}}{W_{1}-W_{3}}
$$

It is helpful to write Eq. (28) in terms of material coefficients $Q_{i j}$ as

$$
\hat{D}_{66} \approx \frac{Q_{11}+Q_{22}-2 Q_{12}}{Q_{11}+Q_{22}+2 Q_{12}+4 Q_{66}}
$$

It has previously been established that both the numerator and denominator decrease with relaxation; however, noticing that

$$
\begin{aligned}
& Q_{11}+Q_{22}+2 Q_{12}+4 Q_{66} \\
& \quad=\left(Q_{11}+Q_{22}-2 Q_{12}\right)+4 Q_{12}+4 Q_{66}
\end{aligned}
$$


it becomes clear that the denominator relaxes faster (by the extra term $\left.4 Q_{12}+4 Q_{66}\right)$ than the numerator. Hence $\hat{D}_{66}$ increases with relaxation.

In conclusion, the $\hat{D}_{66}$ and $\hat{D}_{12}$ terms increase with relaxation effects, while the $\hat{D}_{22}$ term is approximately constant, which supports the results shown in Fig. 7. Hence, it follows from Eq. (3) that the stability criterion $S$ increases with relaxation, confirming the important result shown in Fig. 8, which is that the structure remains bistable at all times.

\section{Deployment Model}

6.1 Analytical Model. An analytical deployment model, based on the work in Refs. [3] and [7], is used to predict the deployment of the spring. The model, originally formulated for elastic isotropic STEMs, has here been generalized for orthotropic viscoelastic laminates. This model assumes that the structure immediately transitions from a coiled shape to an extended state. In reality, however, the transition occurs smoothly within the ploy region, but this has been neglected. In the following, the $x$-axis refers to the longitudinal direction of the deployed spring, and the $y$-axis to the orthogonal direction, originally defined in Fig. 2.

The bending strain energy in a composite shell of length $x$, deformed such that the curvature change is $\Delta \kappa$ is

$$
U_{b}=\frac{1}{2} \Delta \kappa^{\mathrm{T}} \mathbf{D} \Delta \kappa A
$$

where $A$ is the midsurface area of the shell, given by $A=\beta R x$ [7]. Here, it can be assumed that $\Delta \kappa_{x y}=0$ as twist is sufficiently small. Additionally, because $D_{16}=D_{26}=0$, Eq. (31) reduces to

$$
U_{b}=\frac{1}{2}\left(D_{11} \Delta \kappa_{x x}^{2}+2 D_{12} \Delta \kappa_{x x} \Delta \kappa_{y y}+D_{22} \Delta \kappa_{y y}^{2}\right) A
$$

As the tape spring is deployed, the curvature in the longitudinal direction $\kappa_{x x}$ increases from 0 to $1 / r$, and the curvature in the transverse direction $\kappa_{y y}$ decreases from $1 / R$ to 0 . Therefore, the net curvature changes are $\Delta \kappa_{x x}=1 / r$ and $\Delta \kappa_{y y}=-1 / R$. Factorizing and rearranging yields

$$
U_{b}=\frac{D_{11} \beta x}{2 R}\left(\frac{D_{22}}{D_{11}}-\frac{2 R}{r} \frac{D_{12}}{D_{11}}+\frac{R^{2}}{r^{2}}\right)
$$

6.2 Ejection Force. The ejection force $F_{\mathrm{e}}$ is the force available for deployment of the spring. $F_{\mathrm{e}}$ is equal to the restraining force needed to keep the spring at equilibrium during deployment. $F_{\mathrm{e}}$ is given by the rate of change of the strain energy with respect to the extension [3]; hence,

$$
F_{\mathrm{e}}=\frac{\partial U_{b}}{\partial x}
$$

A correction for nonsymmetric laminates is applied using the reduced bending stiffness $\mathbf{D}^{*}$ [11]. Letting the bending stiffness constants vary with time, the ejection force is given by

$$
F_{\mathrm{e}}(t)=\frac{D_{11}^{*}(t) \beta}{2 R}\left(\hat{D}_{22}^{*}(t)-\frac{2 R}{r} \hat{D}_{12}^{*}(t)+\frac{R^{2}}{r^{2}}\right)
$$

It is assumed that the ejection force does not change during deployment, as the deployment occurs over a much shorter time scale than relaxation; hence, the ejection force at the end of relaxation is taken as the input for the deployment model, so that $F_{\mathrm{e}}=F_{\mathrm{e}}\left(t=t_{\text {rel }}\right)$, where $t_{\text {rel }}$ is the total relaxation (stowage) time.

The variation of the ejection force with stowage time can be simulated, to predict how the ejection force would decrease with stowage time. This behavior is shown in Fig. 9 for stowage at $60{ }^{\circ} \mathrm{C}$ for $3 \mathrm{hrs}$.

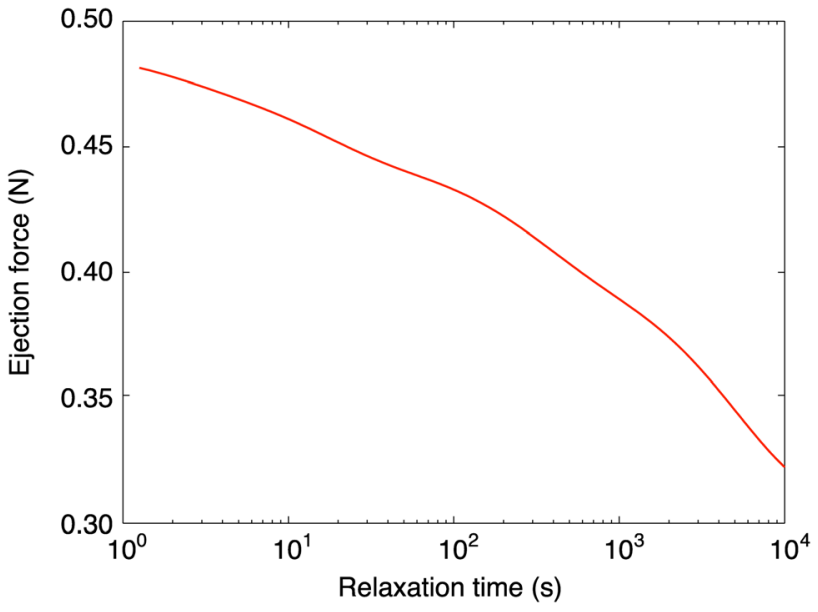

Fig. 9 Plot of the ejection force with stowage time for relaxation at $60^{\circ} \mathrm{C}$. The ejection force available for deployment decreases as the structure relaxes during stowage.

6.3 Aerodynamic Correction. For deployment in air, a simple aerodynamic drag model is implemented to adjust the net ejection force. The drag of the structure is given by

$$
F_{\text {aero }}(t)=\frac{1}{2} \rho v^{2}(t) C_{D} A
$$

The frontal area $A$ is roughly equal by the projected surface of the coiled spring and is given by $A=2 \beta R r$. The drag coefficient $C_{D}$ is based on the average Reynolds number Re. For this application it can be shown that $\mathrm{Re} \approx 1000$, so $C_{D} \approx 1.17$ [18]. As the drag acts in opposite sense to the deployment direction, the corrected ejection force becomes

$$
F_{\mathrm{e}}^{\prime}(t)=F_{\mathrm{e}}-F_{\text {aero }}(t)
$$

6.4 Ejection Velocity. The present configuration is a particular case of a variable mass dynamics problem. Although many more rigorous treatments can be found in the literature, a simple solution to the problem is presented here, inspired from Ref. [3], based on an energy conservation approach.

The kinetic energy of the tape spring $K$ is given by a translating component $K_{\mathrm{t}}$ and a rotating component $K_{\mathrm{r}}$

$$
K=K_{t}+K_{r}
$$

Assuming the coiled radius $r$ does not change during deployment, then

$$
K=m(L-x) \frac{v^{2}}{2}+m(L-x) r^{2} \frac{\omega^{2}}{2}
$$

where $v$ is the ejection velocity and $\omega$ is the angular speed, and $m$ is the mass per unit length. As $\omega=v / R$, Eq. (39) simplifies to

$$
K=m(L-x) v^{2}
$$

Rimrott showed that a frictional force $R$ diminishes the ejection force available for deployment. Friction does indeed occur during the deployment process, e.g., as a result of coils sliding across each other and friction with the deployment surface. The frictional force can be written as a Coulomb force $F_{\mathrm{f}}=\mu F_{\mathrm{e}}^{\prime}$, where $\mu$ is a friction coefficient. The empirical value $\mu \approx 0.6$ was found to be appropriate [3]. By setting the net ejection force $F_{\mathrm{e}}^{\prime}(1-\mu)$ equal to the kinetic energy (assuming that the work done by the force $F_{\mathrm{e}}^{\prime}-F_{\mathrm{f}}$ is fully converted into kinetic energy), then 


$$
F_{\mathrm{e}}^{\prime}(t)(1-\mu)=m(L-x) v^{2}
$$

The ejection velocity is therefore given by

$$
v(t)=\sqrt{\frac{F_{\mathrm{e}}^{\prime}(t)(1-\mu) x}{m(L-x)}}
$$

A simple iterative method is then used to integrate the velocity to obtain the position of the tape spring with time.

\section{Experimental Methods}

7.1 Manufacturing Techniques and Issues. The laminate is assembled on a flat surface then molded onto a cylindrical mandrel. Surface wrinkles can appear on the laminate due to the preforming process and the crumpling of the exterior bag under the applied vacuum. It was found that this effect represents a major source of experimental error as the presence of wrinkles can locally increase the laminate thickness by more than $100 \%$. As such, large variations arise in the local bending stiffness as well as the coiled radius, which can affect the deployment of the spring considerably. To mitigate the appearance of wrinkles, the mandrel is inserted into a shrink-plastic tube. Shrinking of the tube is performed prior to cure using a heat gun. Comparison of the tape springs with and without the shrink tube shows a considerable improvement in the surface finish. Breather fabric and a vacuum bag are then fitted around the mandrel and sealed (Fig. 10). Finally, the laminate is cured at $120^{\circ} \mathrm{C}$ for $3 \mathrm{hrs}$ using an ASC aerospace-grade autoclave.

Although an antisymmetric layup was chosen to reduce bendtwist effects, the resulting structure still presents a significant amount of twist (around $140 \mathrm{deg} \mathrm{m}^{-1}$ after demolding). This effect is due to residual thermal strains appearing in the laminate upon cool-down as a result of the layup antisymmetry. The overall small thickness of the laminate further accentuates this effect.

Although the twisting in the structure is noticeably large immediately after demolding, it reduces significantly over time, settling to around $50 \mathrm{deg} \mathrm{m}^{-1}$ in ambient conditions. In Fig. 10(b), the specimen on the left, demolded immediately after cure, shows a more pronounced twisting than the specimen on the right, which was left to rest for several days. The decrease in twist is most likely due to moisture ingress into the laminate which counteracts the thermal stresses, as observed in Ref. [19].

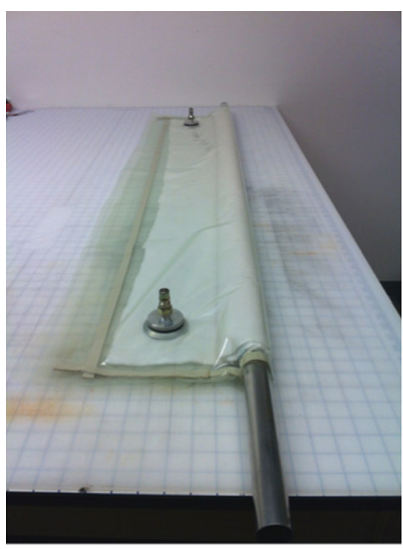

(a)

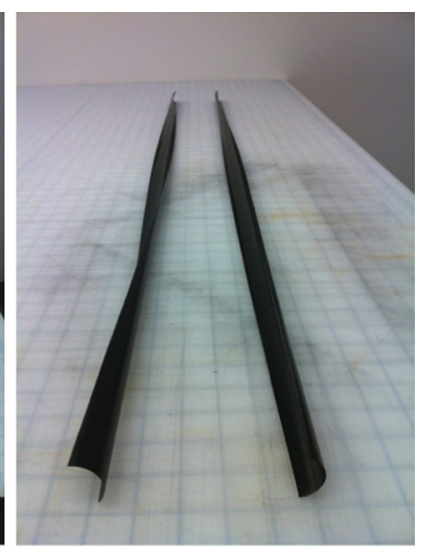

(b)
Fig. 10 Manufacturing technique and demolding issues. (a) The tape spring rests on a cylindrical mandrel and is vacuum bagged prior to cure in autoclave. (b) Twisting of tape springs is observed after demolding, decreasing in magnitude after exposure to air.
7.2 Deployment Apparatus. Three stowage cases are investigated: zero stowage time, stowage for $3 \mathrm{hrs}$ at $60^{\circ} \mathrm{C}$, and stowage at $100^{\circ} \mathrm{C}$. Stowage consists of coiling and stowing the specimen in the Instron thermal chamber for $3 \mathrm{hrs}$ at the set temperature. A thermocouple is used to monitor the chamber temperature. At the end of the stowage period, the structure is removed from the chamber and deployed at room temperature $\left(20^{\circ} \mathrm{C}\right)$ on a flat, horizontal surface. This procedure is followed because it is impractical to perform the deployment within the thermal chamber. A camcorder is used to record the deployment of the tape spring at a frame rate of 29 frames per second. The position of the center of the coil for each time frame is then determined using a video editor.

Previous research showed that there is no significant difference in deploying the structure at room temperature rather than at the temperature at which it has been stowed [20]. This result is expected mainly because the deployment occurs over a much shorter time scale than the relaxation. Hence, it is possible to neglect viscoelastic effects during deployment.

\section{Deployment Results}

8.1 Deployment Without Stowage. Stills taken from the deployment video show a rapid and generally smooth unrolling of the structure (Fig. 11). However, near the end of deployment (between frames Figs. 11(c) and 11(d)), the assumption of uniform uncoiling breaks down. In this region, the tape spring starts to unravel laterally, followed by a dynamic latching of the tip. Therefore, it is convenient to neglect the final uncoiling of the structure, and the deployment time is henceforth defined as the time it takes to achieve $90 \%$ of the full deployment.

Figure 12 compares the analytical (predicted) and experimental (measured) deployment curves for both cases: without and after stowage at $60^{\circ} \mathrm{C}$ for $3 \mathrm{hrs}$. The curves confirm that the overall deployment is smooth and uniform, and that the rate of deployment increases with time. The analytical curves are shown with bounds corresponding to $\mu=0.55$, and $\mu=0.65$, indicating the sensitivity of the analysis to the choice of the friction coefficient.

The experimental results are used to validate the analytical model for the case without stowage. Here, the experimental results match closely the model predictions for $\mu=0.6$. The analytical model predicts a deployment time of $0.41 \mathrm{~s}$, compared with $0.40 \mathrm{~s}$ in the experiment, i.e., a $2.5 \%$ difference in deployment time. This suggests that the choice of the empirical parameter $\mu$ is appropriate.

8.2 Deployment After Stowage. When stowage is applied, the curves exhibit a marked decrease in the deployment rate and an increase in the deployment time. Indeed, Eq. (35) shows that a decrease in the ejection force due to relaxation causes a drop in the deployment velocity, which is the effect observed here.

The analytical results for the stowed configuration do not match the experimental results as well. For the case $\mu=0.6$, the analytical model visibly underestimates the deployment time, with the analytical model predicting deployment in $0.52 \mathrm{~s}$, while

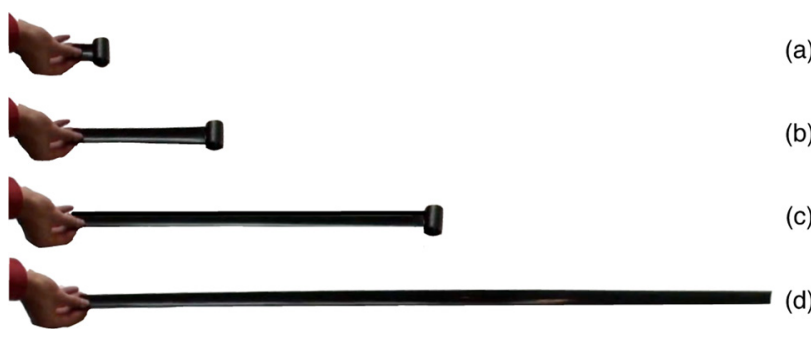

Fig. 11 Snapshots of the deployment of the tape spring with zero stowage time, with (a) $t=0.10 \mathrm{~s}$, (b) $t=0.28 \mathrm{~s}$, (c) $t=0.38 \mathrm{~s},(d) t=0.42 \mathrm{~s}$ 


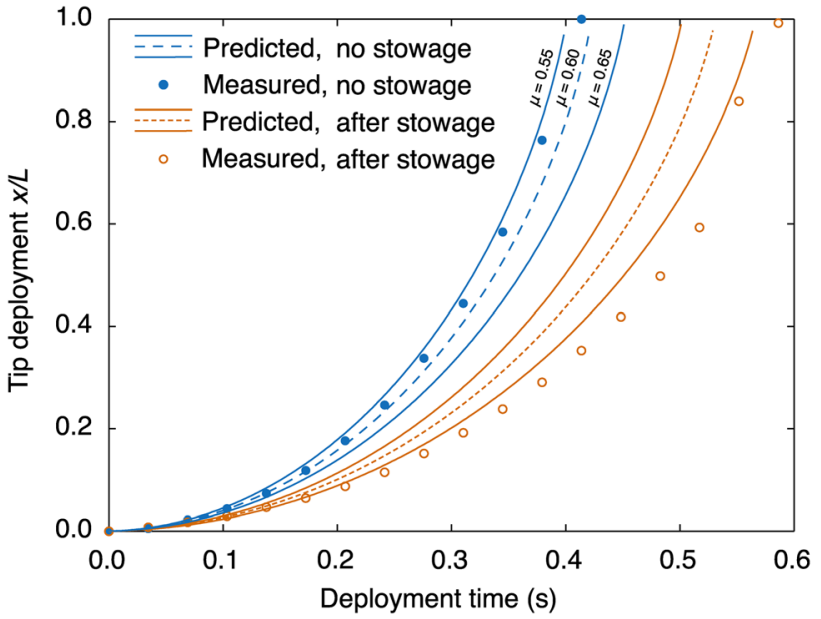

Fig. 12 Comparison of the deployment without stowage and after stowage at $60^{\circ} \mathrm{C}$ for $3 \mathrm{hrs}$, and sensitivity of the analysis to changes in the $\mu$ friction parameter. Stowage of the structure causes an increase the deployment time.

the experiment shows a deployment in $0.57 \mathrm{~s}$ (i.e., an underestimation of $9 \%$ ).

The underestimation of deployment time in the stowed configuration suggests that the ejection force is in fact less than that predicted. This could be due to errors in material characterization, or how the Prony series approximation is represented. To verify this hypothesis, the analytical model is repeated for slightly different viscoelastic representations of the material, by performing a sensitivity analysis on the Prony coefficients. This analysis, shown in Fig. 13, confirms that increasing the relaxation of the material tends to increase the deployment time of the tape spring. It is noticed that the curve for an additional relaxation of $15 \%$ shows the analytical curve approaching the experimental results.

Although an error in material characterization is possible, the observed discrepancy may also suggest that a refinement of the dynamic model is required. In the current model a single coefficient of friction $\mu$, similar to Coulomb friction, is used to represent the loss of ejection force. Further experimental work would be needed to understand better the role of frictional forces-or other dissipative mechanisms - in the deployment of the tape spring.

\section{Secondary Effects}

9.1 Deployment Latency. The previous results have shown that the stowage of the tape spring leads to an increase in the deployment time. In most of the cases observed until now, the deployment remains continuous and consists of a rapid dynamic deployment of the spring once the constraint has been released.

It has been observed, however, that in certain cases the deployment of the tape spring is not continuous and consists of two distinct behaviors. The first is a latent region where no or little deployment is observed, even though the tape spring is no longer restrained. The second is the normal smooth deployment observed previously. Figure 14 shows the deployment curve for a tape spring stowed at $60{ }^{\circ} \mathrm{C}$ for $3 \mathrm{hrs}$ for this type of deployment. The latent region lasts in this case for around $0.45 \mathrm{~s}$, before the spring deploys dynamically in $0.55 \mathrm{~s}$. Total deployment lasts around $1.0 \mathrm{~s}$, which is nearly twice as much as the dynamic deployment shown in Fig. 14. The dynamic part of the deployment is identical for both types of deployment. However, the latent region is subject to typically large variations, making it difficult to predict the total deployment time.

The fact that this latent behavior appears only in tape springs that have been subject to long storage times and high temperatures suggests that viscoelastic relaxation effects are influential. These

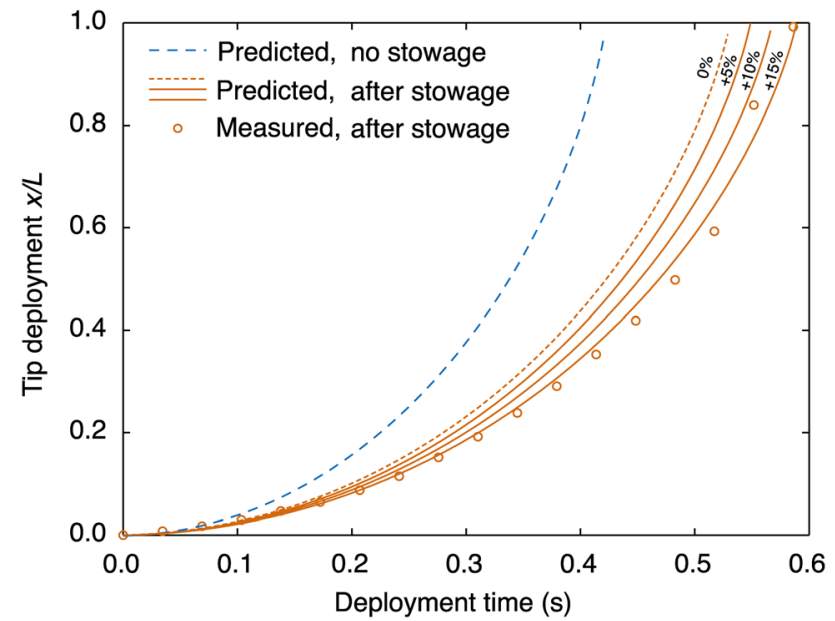

Fig. 13 Sensitivity of the deployment analysis to a change in relaxation of the material. The curves $5 \%, 10 \%, 15 \%$ represent the additional relaxation, compared to the behavior obtained in Table 3.

secondary latency effects, which may arise from local effects in the transition zone, are not captured by the analytical model.

It is hypothesized that the enhanced stability predicted in Fig. 8 can be connected to the observed latency effect. It is suggested that additional stability states between the fully coiled and uncoiled states, due to structural and material variability, are made evident during relaxation. As was shown, relaxation tends to deepen these stability wells. This could explain why these secondary stability states start to influence the response during relaxation and lead to the tape spring sitting idle for a period while it slowly begins to unwind. As the geometry changes and partial recovery of the modulus occurs, the structure finally begins to deploy.

Finally, it is suggested that the experimental deployment technique is refined to eliminate effects due to manual release of the spring, and a gravity offloading rig is used to minimize error due to frictional and gravitational forces.

9.2 Deployment Failure. The stowage of the tape spring at $100^{\circ} \mathrm{C}$ for $3 \mathrm{hrs}$ represents a singular case. When stowed in these

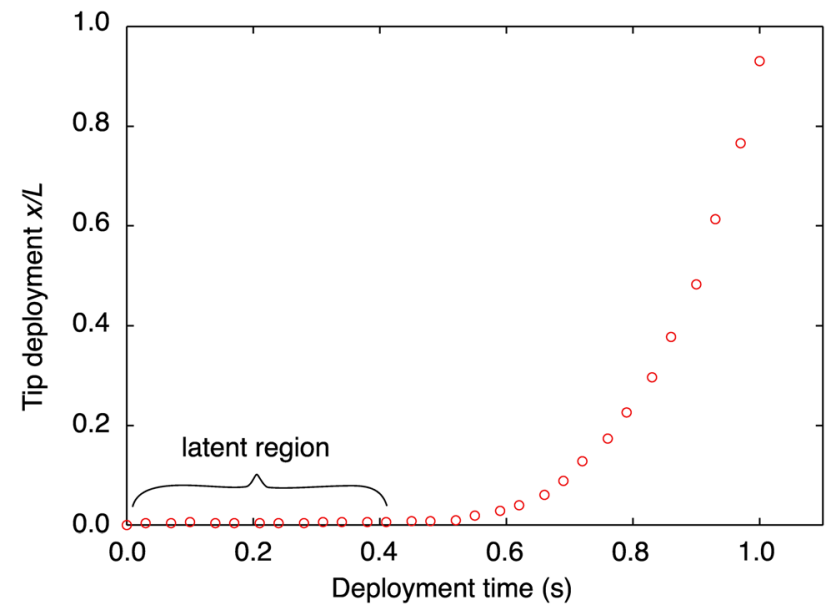

Fig. 14 Deployment after stowage at $60^{\circ} \mathrm{C}$ for $3 \mathrm{hrs}$. Deployment here consists of a latent region with little or no deployment, immediately followed by full deployment. The latent region significantly increases the total deployment time; here the total deployment time is double that of a normal deployment. 


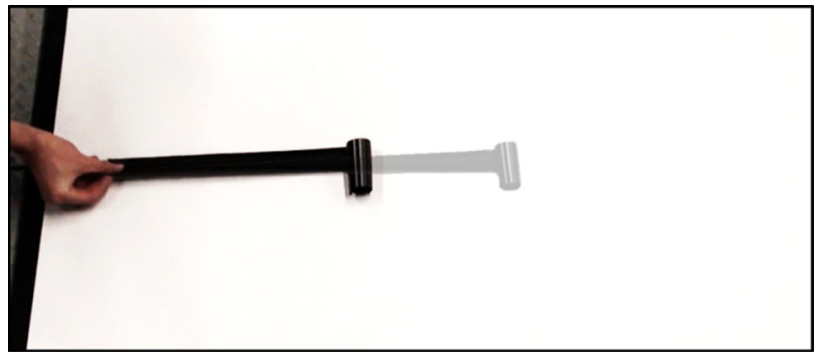

Fig. 15 Photographs of the spring overlaid at various deployment stages. The spring remains stable at any extended position.

conditions, the tape spring fails to deploy. Figure 15 shows an overlay of photographs of the deployment of the spring after stowage at $100^{\circ} \mathrm{C}$ for $3 \mathrm{hrs}$. The spring is found to be stable at any extended position (including, of course, the fully deployed position). This observation is coherent with the results of the stability model, because the structure remains bistable and does not autonomously unfurl when coiled. However, contrary to the results of the analytical model, these experiments suggests that the net ejection force of the structure has dropped to zero, and that the structure does not have sufficient stored strain energy for an autonomous deployment. Overall, the deployment failure case could be a catastrophic outcome if the sole function of the tape spring were to be autonomous deployment. Mitigating solutions, such as the use of a deployment actuator, therefore need to be implemented, if this stowage condition is expected to arise.

Previous research has experimentally found a similar behavior when the matrix stiffness was selected to be low enough [21]. This suggests that it is indeed matrix relaxation that is responsible for the deployment failure of the tape spring. However, the current analytical model is not able to capture at present this important change in behavior and should be modified in future work.

9.3 Deployment Repeatability. Due to experimental constraints, only one tape spring prototype was used for the deployment tests. The test for the case of deployment without stowage was performed several times on consecutive days to test the repeatability of the tests (Fig. 16). To allow for recovery, the spring is uncoiled and stowed at room temperature for one day. Results reveal that the deployment time increases with the number of the tests, and seems to settle at an asymptotic value.

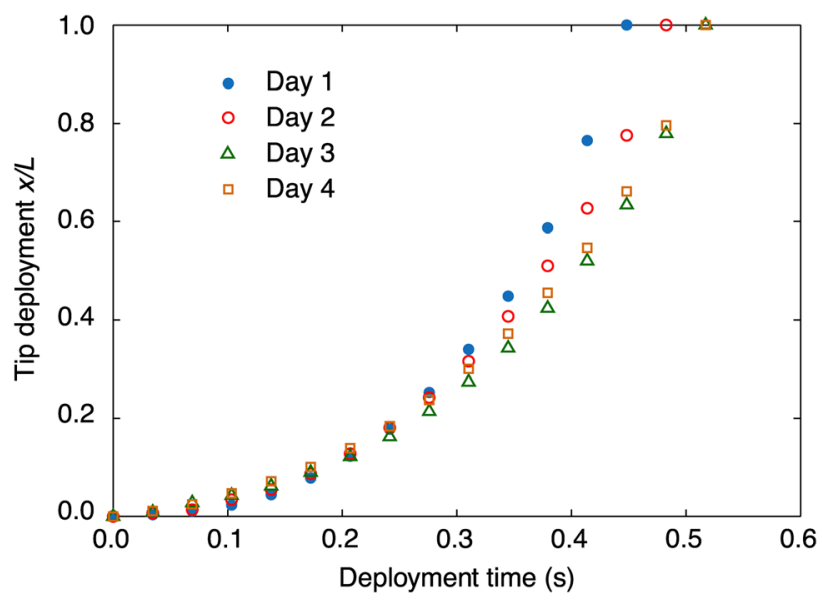

Fig. 16 Comparison on deploying the structure without stowage on successive days. The deployment time increases with the "age" of the specimen. This behavior is most likely attributed to hysteresis effects. It is noted that the days are not consecutive.
These results suggest that the structure experiences some hysteretic effect. Even if the spring is allowed to rest between successive tests to reduce the hysteretic effect, the results show that this is insufficient to restore the initial stiffness completely, which results in a residual relaxation at the start of the next test. This effect grows at higher temperatures, because the structure would experience more relaxation.

This study has informed the test procedure for subsequent tests, and the prototype was allowed to rest for at least one week prior to testing at $60^{\circ} \mathrm{C}$ and $100^{\circ} \mathrm{C}$. Because it is impossible to know the state of residual relaxation without actually performing a deployment test, it is possible that the tests at $60^{\circ} \mathrm{C}$ and $100^{\circ} \mathrm{C}$ contain residual relaxation from the tests at room temperature. This may explain the accrued discrepancy in the deployment time observed for the experimental deployment after stowage at $60^{\circ} \mathrm{C}$ for $3 \mathrm{hrs}$.

Moisture may also have some effect on the deployment behavior. It has been observed that the residual twist in the laminate reduces considerably with exposure time to ambient air, which was attributed to moisture ingress. The resulting change in the geometry may then in turn influence the recovery behavior to a slight extent. Therefore, it is unclear how hysteresis, environmental, or ageing effects precisely affect the deployment of the spring. This could be the object of future work.

\section{Conclusions}

In conclusion, relaxation effects due to stowage of the tape spring in a coiled state cause significant changes in the deployment behavior of the structure. In particular, stowing the structure at higher temperatures or for longer periods of time increases the dynamic deployment time of the structure by decreasing the stored strained energy available for deployment. In cases where the relaxation of the structure is significant, secondary deployment effects have been shown. For instance, stowing the structure at $60^{\circ} \mathrm{C}$ for $3 \mathrm{hrs}$ leads to a latency effect-i.e., a significant delay prior to the dynamic deployment of the boom. This effect is thought to arise from local stability effects, which are not captured at present by the analytical model. For stowage at $100{ }^{\circ} \mathrm{C}$ for $3 \mathrm{hrs}$, the tape spring fails to deploy and remains stable at any deployed position. This effect has been linked to a significant reduction in the matrix stiffness, effectively resulting in a zero ejection force.

The deployment failure of the tape spring for high stowage configurations represents a clearly undesirable effect. In this case, the tape spring loses its ability to autonomously deploy, which would be catastrophic for missions that rely solely on this behavior. Yet, the tape spring still retains a structural function. Provided an actuator is used to achieve full deployment, the tape spring would still be able to latch into its fully deployed state and resist, albeit to a lesser extent, inertial and vibrational forces. The reduction in stored energy could actually be used at an advantage to eliminate dynamic latching effects, which in turn reduces the risk of structural failure and increases the reliability of the deployment. Moreover, such a structure needs minimal energy for deployment, simplifying the design of payout mechanisms and leading to smaller and lighter actuators.

\section{Acknowledgment}

A.B. and P.M.W. would like to thank the EPSRC for the financial support of the project under the ACCIS Centre for Doctoral Training (EP/G036772/1). The authors thank John Steeves and Keith Patterson (Caltech) for their help with experimental methods, and Petros Arakelian (Caltech) for technical advice and support.

\section{References}

[1] Mobrem, M., and Adams, D., 2009, "Deployment Analysis of the Lenticular Jointed Antennas Onboard the Mars Express Spacecraft," J. Spacecr. Rockets, 46(2), pp. 394-402. 
[2] Kwok, K., and Pellegrino, S., 2010, "Shape Recovery of Viscoelastic Deployable Structures," 51st AIAA/ASME/ASCE/AHS/ASC Structures, Structural Dynamics, and Materials Conference, Orlando, FL, Apr. 12-15, AIAA Paper No. 2010-2606.

[3] Rimrott, F., 1967, "Stem Self-Extension Velocities," Can. Aeronaut. Space J., 13(1), pp. $1-7$

[4] Northrop Grumman, 2013, "Storable Tubular Extendable Member," Astro Aerospace, Northrop Grumman Corp., Carpinteria, CA.

[5] Rolatube, 2013, "Our Business," Rolatube Technology, Lymington, UK, http:// www.rolatube.com/our-business

[6] Seffen, K. A., and Pellegrino, S., 1999, "Deployment Dynamics of Tape Springs," Proc. R. Soc. London, Ser. A, 455(1983), pp. 1003-1048.

[7] Iqbal, K., and Pellegrino, S., 2000, "Bi-Stable Composite Shells," 41st AIAA/ ASME/ASCE/AHS/ASC Structures, Structural Dynamics, and Materials Conference and Exhibit, Atlanta, GA, Apr. 3-6, AIAA Paper No. 2000-1385.

[8] Kwok, K., and Pellegrino, S., 2011, "Viscoelastic Effects in Tape-Springs," 52nd AIAA/ASME/ASCE/AHS/ASC Structures, Structural Dynamics and Materials Conference, Denver, CO, Apr. 4-7, AIAA Paper No. 2011-2022.

[9] Brinkmeyer, A., Pellegrino, S., Weaver, P. M., and Santer, M., 2013, "Effects of Viscoelasticity on the Deployment of Bistable Tape Springs," 19th International Conference on Composite Materials (ICCM-19), Montreal, QC, Canada, July $28-$ Aug. 2 , pp. $370-380$

[10] Steeves, J., 2012, "T800H Room Temperature Elastic Properties," California Institute of Technology, CA.
[11] Mansfield, E. H., 2005, The Bending and Stretching of Plates, 2nd ed. Cambridge University Press, Cambridge, UK.

[12] Matlab, 2010, Matlab User Guide, 7.10 ed., The Mathworks, Inc., Natick, MA.

[13] Ferry, J. D., 1980, Viscoelastic Properties of Polymers, Vol. 3, Wiley, New York.

[14] Hull, D., and Clyne, T. W., 1996, An Introduction to Composite Materials, Cambridge Solid State Science Series, Cambridge, UK.

[15] Bazant, Z. P., and Cedolin, L., 2010, Stability of Structures: Elastic, Inelastic, Fracture and Damage Theories, World Scientific, Singapore.

[16] Guest, S. D., and Pellegrino, S., 2006, "Analytical Models for Bistable Cylindrical Shells," Proc. R. Soc., Ser. A, 462(2067), pp. 839-854.

[17] Bloomfield, M., Diaconu, C., and Weaver, P., 2009, "On Feasible Regions of Lamination Parameters for Lay-up Optimization of Laminated Composites," Proc. R. Soc. London, Ser. A, 465(2104), pp. 1123-1143.

[18] Anderson, J. D., 2001, Fundamentals of Aerodynamics, McGraw-Hill, Boston, MA.

[19] Etches, J., Potter, K., Weaver, P., and Bond, I., 2009, "Environmental Effects on Thermally Induced Multistability in Unsymmetric Composite Laminates," Composites, Part A, 40(8), pp. 1240-1247.

[20] Kwok, K., and Pellegrino, S., 2012, "Micromechanical Modeling of Deployment and Shape Recovery of Thin-Walled Viscoelastic Composite Space Structures," 53rd AIAA/ASME/ASCE/AHS/ASC Structures, Structural Dynamics, and Materials Conference, Honolulu, HI, Apr. 23-26, AIAA Paper No. 2012-1910.

[21] Schultz, M. R., Hulse, M. J., Keller, P. N., and Turse, D., 2008, "Neutrally Stable Behavior in Fiber-Reinforced Composite Tape Springs," Composites, Part A, 39(6), pp. 1012-1017. 